\title{
El macrismo ¿neoliberal? Política urbana en el sur de la Ciudad Autónoma de Buenos Aires*
}

\begin{tabular}{|c|c|c|}
\hline \multicolumn{3}{|c|}{ Fecha de recepción: día de mes de año Fecha de aceptación: día de mes de año Disponible en línea: 2 de abril de 2015} \\
\hline María Cristina Cravin & & \\
\hline Doctora en Antropología & Investigadora Conicet & mariacristinacravino@yahoo.com.ar \\
\hline
\end{tabular}

\section{Ariel Matías Palombi \\ \begin{tabular}{l|l|l}
\hline Doctorando en Ciencias Sociales & Becario Conicet & arielpalombi@hotmail.com
\end{tabular}}

Resumen Este trabajo forma parte de una investigación en curso acerca de las políticas de desarrollo urbano y vivienda de la gestión del ingeniero Mauricio Macri (2007-2014) en el sur de la Ciudad Autónoma de Buenos Aires. En el marco de la finalización de su segundo mandato, hacia el año 2015, el presente artículo tiene como objetivo brindar algunos elementos conceptuales para un balance crítico de la gestión. A partir del concepto de "neoliberalismo realmente existente" acuñado por Theodore, Peck y Brenner (2009) se estudian las acciones implementadas por dicha gestión en estos barrios y se construye una tipología de zonas que emergen como producto de las políticas y una periodización de las mismas. Esta investigación en curso se basa en entrevistas a funcionarios del Gobierno de la ciudad, dirigentes barriales y legisladores y en el estudio de fuentes secundarias como documentos legislativos y periódicos nacionales.

\section{Gestión urbana; macrismo; neoliberalismo; zona sur de Buenos Aires}

Este artículo es producto de una investigación finalizada, que llevó por título "La nueva cuestión urbana en el Área Metropolitana de Buenos Aires. Conflictos, demandas y acción pública en torno al hábitat" (PICT 2008 2120), iniciada en abril de 2010 y finalizada en setiembre de 2014. Fue financiada por la ANPCYT, inserta en el área de Urbanismo del Instituto del Conurbano de la Universidad Nacional de General Sarmiento y dirigida por María Cristina Cravino.

Cómo citar este artículo: Cravino, M. C. y Palombi, A. M. (2015). El macrismo ¿neoliberal? Política urbana en el sur de la Ciudad Autónoma de Buenos Aires. Cuadernos de Vivienda y Urbanismo, 8(15), xx-xx. http://dx.doi.org/10.11144/Javeriana.cvu8-15.mnpu 


\section{Macrismo Neoliberal? Urban Policy in the South of Buenos Aires}

Abstract The work is part of an ongoing investigation about the policies of urban development and housing management carried forward by Mauricio Macri (2007-2014),) in the south of Buenos Aires. As part of the completion of his second term to 2015, this article aims to provide some conceptual elements for a critical assessment of management. Based on the concept of actually existing neoliberalism, coined by Neil Brenner, et all (2009), is to studied the various actions taken by management that are discussed in these neighborhoods, building a typology of areas that emerge as a result of policies and a periodization of them. This ongoing research is based on interviews with government officials of the City, neighbourhood leaders, legislators and the study of secondary sources such as legislative documents and three national newspapers.

Keywords Buenos Aires; urban policy; urban neoliberalism

\section{Macrismo ¿neoliberal? políticas urbanas no sul da Cidade de Buenos Aires}

Resumo Este trabalho é parte de uma investigação em andamento sobre as políticas de desenvolvimento urbano e moradia no sul da cidade de Buenos Aires sob o governo do engenheiro Mauricio Macri (2007-2014). O artigo pretende fornecer alguns elementos conceituais para um balanço crítico dessa gestão. Baseado no conceito de "neoliberalismo realmente existente", cunhado por Theodore, Peck e Brenner (2009) são investigadas as açóes realizadas nestes distritos e se constrói uma periodização e uma tipologia das áreas conforme essas políticas. Para esta pesquisa foram utilizadas informaçóes primárias, tais como entrevistas com funcionários do governo da cidade, lideranças políticas e sociais e também informações secundárias de jornais nacionais e documentos do legislativo.

Palavras chave Cidade de Buenos Aires; política urbana; neoliberalismo urbano 


\section{Introducción}

El siguiente artículo se propone analizar las heterogéneas políticas urbanas y proyectos que se desarrollaron en el sur de la Ciudad Autónoma de Buenos Aires durante la gestión del jefe de Gobierno Mauricio Macri, en el período 20072013, quien finalizará su segunda gestión en el año 2015. Si bien su partido, Propuesta Republicana (PRO), es caracterizado por los cientistas políticos como de derecha, este adjetivo no expresa a cabalidad lo que sucede en sus intervenciones urbanas, ya que su impronta pragmática, de constantes iniciativas y prácticas diferenciales, obliga a hacer un análisis más atento a sus matices y cambios a lo largo de sus dos gestiones. Estas acciones han generado marcas simbólicas en los barrios, en clave de fragmentos urbanos, a la vez que se encuentran en un marco de proyectos de mayor alcance territorial.

En primer lugar, se expondrá la conformación histórica del sur de la ciudad y su situación actual, como una primera aproximación a la dimensión política del macrismo y luego revisaremos las políticas, los proyectos y las intervenciones urbanas, en tanto dimensiones contradictorias, contrastantes y de fuertes efectos en el orden y las jerarquías de la ciudad.

\section{Buenos Aires al sur: integración y abandono}

A partir de fines del siglo XIX, en la Ciudad Autónoma de Buenos Aires se desarrolló una tendencia a una fuerte diferenciación social y urbana en detrimento del sur. De esta manera, la avenida Rivadavia ha sido el eje geográfico y simbólico que ha dividido a la ciudad en dos grandes territorios. La zona sur está constituida por tres comunas completas $(4,8,9)$ y sectores de otras tres $(1,3 \text { y } 7)^{1}$.

La postergación de la zona sur respecto a la norte ha sido un tópico de debate permanente a partir de la década del treinta. Se han encontrado diversos planes y propuestas, algunas solo en el marco de campañas electorales y otras alcanzaron mayor desarrollo. La zona sur es conocida por presentar los peores indicadores sociourbanos de la ciudad de Buenos Aires. Es el espacio donde se concentra el déficit en oferta pública, vivienda, salud y educación y se caracteriza por la presencia de diversas tipologías residenciales: casas bajas, inquilinatos, viviendas ocupadas (Herzer, 2008; Di Virgilio y Rodríguez, 2013), conjuntos habitacionales en altura construidos por el Estado (Girola, 2005) y villas (Cravino, 2007). Estas últimas dos formas adquieren una mayor presencia que en el norte de la ciudad y le dan una impronta particular.

En su historia convergen varios procesos, caracterizados por la instalación de diferentes actividades económicas (fabriles, mercados de hacienda, etc.), la implantación de conjuntos habitacionales para sectores populares, de estrategias habitacionales autogestivas y, en menor medida, del desarrollo inmobiliario privado.

1 La comuna 1 corresponde al área central, por lo que escapa a los planteamientos referidos a la zona sur como espacio de intervención urbana. 
Frente a la idea de un "sur" homogéneo, como una y otra vez pareciera aludirse en las propuestas o en el debate público, es necesario visualizar distintos modos de conformación urbana, tipologías residenciales, sociabilidades y jerarquías urbanas. A grandes rasgos, existe una notoria diferencia de trayectoria histórica entre dos grandes grupos de barrios, en particular entre La Boca, Barracas, Balvanera, Parque Patricios y Pompeya respecto a Villa Lugano, Villa Soldati y Bajo Flores. El primero se destacó tempranamente por el desarrollo de usos industriales, combinado con zonas de grandes equipamientos urbanos y vivienda, entre los que vale mencionar grandes hospitales, estaciones ferroviarias, fábricas y depósitos que hasta hace algunos años se encontraban, en muchos casos, en situación de abandono. En estas comunas existe suelo vacante o perteneciente a grandes equipamientos, que puede ser utilizado para desarrollar emprendimientos privados o procesos de recualificación ${ }^{2}$. El segundo responde más al modelo de "ciudad dormitorio" o autoconstruida, que hasta la década del setenta se caracterizó por una cantidad significativa de espacio vacío, al tratarse de terrenos inundables (bañados) o basurales, incluido el basurero municipal ${ }^{3}$.

Esto dio lugar a que esta zona, correspondiente a las actuales comunas 7 y 8 , posea un carácter "intersticial, en las que coexisten espacios desarticulados y fragmentados, a pesar de la continuidad física que puede existir entre ellos" (Perelman, 2011, p. 7). Desde una perspectiva histórica, es importante resaltar la importancia del Estado en la zona como sujeto de ordenamiento territorial, por medio de la construcción de viviendas de interés social (sobre todo en altura), dado que el mercado le ha asignado poca importancia a la construcción residencial. En términos de Marcuse (2004), en esta zona el Estado ha cumplido un rol importante en la diferenciación urbana y división social del espacio ${ }^{4}$.

Durante la última década, en la Ciudad Autónoma de Buenos Aires se ha consolidado una tendencia a que el patrón de distribución territorial de la población sea inverso al patrón de valorización del suelo. En la década 2000-2010, la oferta privada de viviendas se ha expandido a razón de $1.332 .939 \mathrm{~m}^{2}$ por año, con un total de 14.662.327 m² (Cosacov y Faierman, 2012). Aunque desde el año 1947 la ciudad posee casi la misma cantidad de población cercana a los 3.000.000, si se compara el censo 2001 con el censo 2010 la población de la ciudad se incrementó en un 4,1\%. Este crecimiento se explica por el aumento de población en el sur de la ciudad (en la comuna 8, en especial), mientras en la zona norte, en donde se registró la mayor cantidad de permisos de construcción, se aprecia un estancamiento e incluso, en algunas comunas, decrecimiento de población. De esta manera, las zonas de mayor valor del suelo y construcción no son las que más población atraen ${ }^{5}$.

Al mismo tiempo, la evolución de la situación habitacional en la CABA en el período intercensal (2001-2010) muestra un empeoramiento de las condiciones habitacionales. Aumentaron

\footnotetext{
2 Cabe recordarse la política de desindustrialización desarrollada durante la última dictadura militar (1976-1983) y la expulsión de la industria hacia la periferia urbana Para un desarrollo pormenorizado de la cuestión puede consultarse Cosacov y otros autores (2011)

4 Entre las comunas que se encuentran en la zona sur, la 8 (Villa Lugano, Villa Riachuelo y Villa Soldati) se destaca por poseer la mayor presencia de población que habita en villas. Así, mientras el promedio de la población que vive en villas en el conjunto de la ciudad es de 5,7\%, en este distrito es de 32,9\% (Instituto de Estadísticas y Censos, 2010). Entre los asentamientos informales se destacan, la 15 "Ciudad Oculta" y Núcleo Habitacional Transitorio Avenida del Trabajo, Villa 17 Barrio "Pirelli", Villa 19 Barrio "INTA" y Villa 20 "Lugano". Esta última villa es la segunda en importancia de la Ciudad Autónoma de Buenos Aires, por la cantidad de población que concentra. El aumento de los habitantes de este tipo de barrios en dicha comuna determinó también que fuera la de mayor incremento intercensal de población de la ciudad (22,3\% entre 2001 y 2010), al tiempo que la mayoría de las comunas decrecieron en población.

5 La superficie para multiviviendas representa un 90\% de la superficie residencial construida entre 2000 y 2010 (Cosacov y Faierman, 2012).
} 
las personas que viven en las villas y las que viven alquilando piezas en inquilinatos, pensiones $\mathrm{u}$ hoteles, a la vez que hay un incremento de los hogares que residen en viviendas que no son deficitarias, pero que tienen importantes grados de hacinamiento. Según el Censo 2010 (Instituto de Estadísticas y Censos, 2010), casi un 6\% (163.587 personas) de la población de la ciudad vive en villas, NHT o asentamientos, lo que implica un aumento de 52\% respecto al año 2011.

Por otra parte, hay 103.963 personas (45.906 hogares) que residen en piezas de inquilinatos, hoteles o pensiones, localizados en mayor medida en las comunas 1, 3, 4 y 7. Esto indica un crecimiento significativo frente a 2001, que registró la presencia de 39.434 hogares que vivían bajo esa modalidad. También vale destacar que la falta de oportunidades para la compra, debido al aumento del precio del suelo por encima del promedio de inflación y del alza de salarios, se refleja en los datos del último censo. Se ha producido un acrecentamiento de los hogares inquilinos de 51\% en relación con 2001 .

Este empeoramiento de las condiciones urbanas y del acceso a una vivienda se traduce en mayor conflictividad urbana (resistencia a desalojos, ocupaciones de tierra en zonas intersticiales, manifestaciones por el acceso a una vivienda o a un subsidio habitacional, reclamos de créditos a cooperativas en el marco de la Ley 341, etc.), que ha cobrado importancia en la escena pública $^{6}$ por medio de distintos repertorios de acción colectiva. Si bien estas demandas implican una mayor visibilización de la zona sur, también significan formas de estigmatización. Los medios de comunicación presentan a la zona sur como "insegura", "pobre” y "deteriorada”.

\section{El macrismo: una derecha heterodoxa}

El PRO es un partido político de origen reciente, que emergió en la Ciudad Autónoma de Buenos Aires luego de la crisis de 2001. Como bien afirma Vommaro (2013), ha formado sus élites dirigentes con base en una importante cantidad de cuadros que no venían del mundo de la política. Para el autor, se trata de un partido pragmático, de clases medias altas, que combina una presentación en términos de nueva política con la participación de políticos profesionales de larga data.

Como sostiene Harvey (2008), un notable foco de ascenso de poder de clase bajo el neoliberalismo debe atribuírsele a los altos directivos, que son los operadores decisivos en los consejos de administración de las empresas y a los jefes del aparato financiero, legal y técnico. Podemos notar que, aunque muchos funcionarios del gobierno de Mauricio Macri tienen como antecedente haber ocupado cargos gerenciales en empresas privadas, algunos de ellos mostraron serias limitaciones en su capacidad de gestión, por lo que debió recurrirse al reclutamiento de dirigentes con experiencia política provenientes del Radicalismo, del Partido Justicialista o, en su defecto, de ONG de orientación centro-derecha. El perfil gerencial era el deseado ya que, desde el discurso del PRO, se asociaba con la eficiencia administrativa necesaria para la gestión de la ciudad, mientras se denostaba la experiencia política al vincularla a la corrupción. Esto cambió por el carácter pragmático de dicho partido político. Por eso, Vommaro (2013) distingue cinco facciones que conforman el macrismo:

6 Si bien el conflicto que tuvo más relevancia pública fue la toma del Parque Indoamericano, también formaron parte de la misma serie durante el año 2010 La veredita (desarrollado en torno a la ocupación de aceras de un club en el Bajo Flores), Club Albariño y las viviendas sociales tomadas de la calle Castañares y Lafuente (también en el Bajo Flores). En los años anteriores podemos encontrar el conflicto de Deportivo Español, Villa Cartón y otros de menor magnitud. 
peronistas, radicales, derecha liberal, empresarios y profesionales del mundo de las ONG y fundaciones. Cada una de estas tiene cierta división del trabajo y orienta nuevos sentidos dentro de la política pública.

Ahora bien, lo que nos interesa postular en este artículo es que la política de desarrollo urbano y vivienda llevada adelante por la gestión PRO debe estudiarse en su unidad, en relación con el modelo de ciudad que propone y los entramados sociales urbanos sobre los que se despliega. Creemos que existe un proyecto de ciudad excluyente, donde no siempre la política pública está caracterizada por la retirada del Estado o el vaciamiento de lo público bajo la lógica del neoliberalismo más clásico, sino mediante formas más complejas y cotidianas, en las que prima una tendencia hacia la expulsión de los sectores populares de la ciudad, no solo por coerción violenta, sino también por el mercado.

Por esta razón nos distanciamos de quienes han postulado que las políticas públicas desarrolladas durante el período 2007-2013 apuntan a una retirada del Estado. Por lo contrario, consideramos que la gestión de Mauricio Macri ha significado una reconfiguración del Estado en el distrito analizado.

Resulta interesante observar que a lo largo de la gestión ha habido cambios significativos en la orientación de las políticas a partir de diversos conflictos que se fueron suscitando. Si bien en un principio (2008-2010) el macrismo se caracterizó por una serie de iniciativas de "limpieza social" del espacio público mediante amenazas a los indigentes, con una fuerza de choque especialmente creada para ese efecto, y el desalojo de recicladores urbanos, ambas medidas fueron dejadas de lado tras procesos de judicialización y el rechazo de la opinión pública a la violencia. Desde 2011 hasta la actualidad hubo intentos de "limpieza social" que no llegaron a efectivizarse, como la prohibición de la presencia de los cuidacoches en la vía pública (llamados popularmente "trapitos") y otros con un resultado parcial (2011-2013), como la expulsión de los vendedores ambulantes de las calles y veredas. Al inicio de la gestión también apelaron a la penalización de las protestas en el espacio público, dispositivo que luego utilizaron de forma más acotada, pero que nunca abandonaron. Cabe resaltar la capacidad de la gestión de reorientar su política, al dosificar este tipo de iniciativas en situaciones puntuales. También se deben mencionar acciones de represión por medio de la Policía local (denominada Metropolitana, por esta gestión) en diferentes situaciones, como la ocupación del parque Indoamericano (2010), con dos muertes asociadas con esta Fuerza, una intervención en un hospital neuropsiquiátrico (2013), el parque Centenario (2013) y un teatro (2013).

Una de las principales conclusiones a las que podemos llegar tras siete años de gestión es que el macrismo centró su política urbana en intervenciones en el espacio público. Estas acciones se adelantaron de manera diferenciada: en la zona norte, plazas embellecidas y enrejadas, calles con esquinas reducidas con el fin de impedir el tránsito y los bares con espacio en las veredas; en la zona sur, "maquillaje urbano" (en principio de forma devaluada y luego de mejor calidad) en las villas y acciones en espacios fragmentados, aunque también se desarrollaron políticas de embellecimiento en áreas reservadas para futura recualificación.

Más recientemente surgió una política de transporte público de pasajeros en superficie ante el fracaso de la ampliación de los subterráneos. Consiste en carriles exclusivos para colectivos (con construcción de paradas identificadas y con refugio), que pasaron a llamarse "metrobuses". A su vez, emergió con más fuerza una 
política de búsqueda de espacios acotados y diferenciados de recualificación en la zona sur, como la propuesta de la villa olímpica en la Comuna 8. Para otros lugares se proponen zonas de transición o contención social, que luego vamos a desarrollar.

Creemos que, dada la capacidad de adaptación y de pruebas de carácter contingente, el Gobierno de Mauricio Macri representa un caso de "neoliberalismo realmente existente" (Theodore, Peck y Brenner, 2009) ${ }^{7}$, en tanto posee una forma compleja — siempre sujeta a ser modificadas en las disputas- que convive con algunas de las formas preexistentes dentro del Estado de la ciudad. De esta manera, como afirman los autores, "los programas neoliberales de restructuración capitalista nunca son impuestos en forma pura, porque se introducen en contextos políticos-institucionales que han sido moldeados significativamente por un legado de disposiciones regulatorias, prácticas institucionalizadas y arreglos políticos transmitidos a través del tiempo" (Theodore et al., 2009, p. 5). Si bien el proceso de neoliberalización del Estado de la ciudad había comenzado antes de la gestión de Mauricio Macri —en especial con el jefe de Gobierno Jorge Telerman, pues intentó construir gobernanza con articulaciones público-privadas-, el PRO representa la consolidación de este proceso.

En definitiva, podemos afirmar que las políticas públicas desarrolladas por la gestión macrista presentan muchos de los rasgos de los gobiernos típicamente neoliberales, entendido el neoliberalismo como un sistema de legitimación con un marcado objetivo de clase (Harvey, 2008). No obstante, esto implica formas de gobernabilidad que se apropian de modos de gestión previos y estrategias flexibles para sostener o restaurar el poder de las élites.

\section{Distintas políticas dentro de una estrategia excluyente}

La caracterización conceptual del macrismo que presentamos en los apartados anteriores se fundamenta en la política concreta que la gestión implementa en los barrios pertenecientes al sur de la ciudad, según sus especificidades. A la vez, pareciera que los funcionarios del PRO constituyen una variable en estrecha relación con el sentido de las políticas de desarrollo urbano y hábitat. Estas acciones son abordadas por dependencias tan distintas como los Ministerios de Espacio Público, Desarrollo Urbano, Desarrollo Económico, Desarrollo Social, la Corporación Buenos Aires Sur y la Secretaría de Hábitat e Inclusión Social, lo que redunda en políticas diversas y hasta contradictorias, cuyo denominador común es que son respuestas contingentes a territorios con distintos entramados sociales y jerarquías urbanas. Por otra parte, expresan intentos de construcción política territorial diferenciados de acuerdo con los distintos actores gubernamentales.

Podemos entender la política hacia cada uno de los barrios de la siguiente manera:

\section{Políticas de recualificación y renovación urbana}

Estas se desarrollan en los barrios de la ciudad que tienen una cantidad importante de suelo vacante por su conformación histórica (La Boca,

Los autores diferencian al neoliberalismo realmente existente de la ideología neoliberal misma, uno de cuyos supuestos es que las fuerzas del mercado operan de acuerdo con leyes inmutable. El concepto de "neoliberalismo realmente existente" destaca la inserción contextual de los proyectos de reestructuración neoliberal y su dependencia de la trayectoria (Theodore et al., 2009). 
Barracas, San Telmo, Balvanera, Parque Patricios y Pompeya). Dentro de esta área podemos encontrar una zona cercana al centro, en la cual se busca promover los desarrollos de oficinas y viviendas pertenecientes a nuevos sectores de clase media (Barracas y La Boca). En el caso particular de La Boca, el impulso a sectores residenciales se mezcla con la implantación del distrito de las artes (Usina del Arte) y algunas obras de mejoramiento, como la "puesta en valor" (pequeńas obras de mejoramiento o embellecimiento) de la calle Necochea y todo el entorno de la Ribera, que implica el intento de consolidar una zona turística-artística. Vale aclarar que tanto en el barrio de La Boca como en San Telmo existieron diversos planes anteriores tendientes a su consolidación en clave de gentrificación, muchos de los cuales fracasaron.

Respecto a Barracas, allí se intenta construir el distrito gubernamental, que implicaría el traslado de las dependencias del Gobierno de la Ciudad de Buenos Aires hacia esta zona de la ciudad. La iniciativa tiene resistencia de diversos actores sociales, dado que su construcción está planificada sobre terrenos pertenecientes al Hospital Neuropsiquiátrico José Borda, sin que el Gobierno de la ciudad indique cuál será el destino del mismo. Además, la iniciativa viola el Código de Planeamiento Urbano y varias normativas de protección histórica. En esta zona se han adelantado numerosas obras de mejoramiento, como el arreglo de veredas, la construcción de bulevares y obras orientadas hacia el espacio público. Es claro que la intención de la política es consolidar una zona de oficinas y viviendas donde se asiente una clase media joven que valore la cercanía a las centralidades.
En parque Patricios y una parte de Pompeya, la gestión ha diseñado el llamado distrito tecnológico. Esta iniciativa promueve la instalación de empresas del rubro, mediante rebajas impositivas y la ejecución de obras de mejoramiento del entorno. Asimismo, los empleados que trabajen en las empresas y se muden al barrio cuentan con líneas de crédito diferenciales y con exención de pago del impuesto distrital durante diez años. Esta estrategia se complementa con la puesta en funcionamiento de dos nuevas estaciones del subte línea $\mathrm{H}$ y recualificación urbana del parque Patricios. ${ }^{8}$. Dentro de esta última zona también existen algunos sectores con políticas orientadas a garantizar cierto grado de gobernabilidad, como el caso de la Villa 21-24, de Barracas, una de las más numerosas de la ciudad.

\section{Políticas de grandes equipamientos urbanos}

Se localizan en la zona de Villa Soldati y Villa Riachuelo. Es un plan en formación que se basa en la readecuación de algunos aspectos de un proyecto ejecutado durante la última dictadura militar (1976-1983). Estas acciones se adelantan en una porción significativa de suelo vacante y se orientan a propiciar un uso recreativo y turístico. Los proyectos que incluye son la readecuación del Microestadio del Parque Roca y del denominado parque de la Ciudad para el desarrollo de la Ciudad del Rock y la construcción de un terminal de ómnibus, la Villa Olímpica y un centro de transferencia de cargas en el parque Roca. La intención de estas políticas en zonas degradadas es generar equipamientos que logren transformar los usos del suelo a usos mixtos (productivos, de transporte y culturales). 


\section{Política de gobernabilidad}

Estas políticas se llevan a cabo en territorios en condición de vulnerabilidad, sobre todo en los barrios en donde se encuentra la mayor parte de las villas de la ciudad, como la 1-11-14, 20, 3, 15, 6, Piletones, Calaza, Calacita y 19. En estos barrios podemos diferenciar dos líneas complementarias en el abordaje de la cuestión. Por un lado, encontramos una serie de intervenciones que se caracterizan por obras de desarrollo de espacios públicos y comunitarios, mejoramiento de servicios, remodelación de fachadas y atención de emergencias. Estas políticas comenzaron a implementarse a partir de 2009 con la Unidad de Gestión de Intervención Social (UGIS), mediante el armado de cooperativas con vecinos de los propios barrios como una manera de articular una red social y política que garantice gobernabilidad en estos territorios, en particular cuando el partido gobernante no tenía arraigo en ellos. A partir del año 2012, esta política convive con la ejecutada por la Secretaría de Hábitat e Inclusión, en particular, la experiencia de los "portales de inclusión” (espacios públicos orientados a lo deportivo y desarrollo de microemprendimientos). Estas intervenciones se instituyeron en asentamientos de gran escala o complejidad como la Villa 1-11-14 (Bajo Flores) y Villa 20, aunque también en otros de menor escala como la $\mathrm{Vi}$ lla 6 (Cildañez). Esto implica una presencia cotidiana de gran cantidad de agentes estatales, lo que muestra que la política macrista no implica una retirada del Estado, sino una presencia territorial de otro tipo. Merece destacarse que este despliegue de agentes se hace sin tener interlocución con los referentes villeros elegidos por los propios vecinos de estos barrios, por lo cual podemos afirmar que es un estilo de construcción política con un discurso que se asienta en la afirmación de la "no-política".
Por otra parte, existen algunas intervenciones acotadas en asentamientos de menor escala, como las Villas 19, 17 y 6, tendientes a la concreción de una forma de reurbanización de los barrios mediante la apertura de calles, la construcción del algunas viviendas y la regularización de dominio del suelo (esta última basada en la implementación de la ley de propiedad horizontal, no coincidente con los criterios de la Ley 148 de 2000, que regula la reurbanización de villas y asentamientos). Esta política se basa en intenciones de recaudación impositiva y la integración de estos espacios en el catastro de la ciudad, sin importar las condiciones urbanas y habitacionales de los habitantes.

También surgieron algunas políticas en respuesta a distintas sentencias judiciales, como el caso de Villa Cartón (que sufrió un incendio y las familias perdieron sus viviendas) y la llamada "causa Mendoza”, cuya sentencia dictó la Corte Suprema de Justicia y obligaba al saneamiento de la cuenca Matanza-Riachuelo. En la ciudad de Buenos Aires se relaciona con la relocalización de hogares que viven sobre camino de sirga del Riachuelo. Si bien el cumplimiento de las sentencias se ha retrasado y complicado (con finales de obras deficientes, asentamiento de viviendas en terrenos presuntamente contaminados, etc.), el Gobierno de la Ciudad Autónoma de Buenos Aires debió construir viviendas de interés social "llave en mano", que en todos los casos son en altura, pese a que la población contaba con residencia anterior en viviendas de baja altura.

\section{Políticas de zonas fronterizas}

Como ya afirmamos, la política planteada por la gestión Macri se encuentra articulada con los entramados sociales y las jerarquías urbanas propias de cada uno de los territorios. Estas políticas 
se desarrollan en zonas que constituyen el último eslabón de la ciudad formal y creemos que emergieron como respuesta al conflicto del parque Indoamericano durante el mes de diciembre de 2010, como una forma de articular un mecanismo no necesariamente coercitivo que logre garantizar gobernabilidad. La modalidad residencial privilegiada sobre la que se configura este tipo de políticas son los conjuntos habitacionales no degradados (barrios Nágera, Samore, Copello, Juan XXI), la zona de Flores Sur, centro comercial de Lugano y ciertas partes de parque Chacabuco.

Algunas obras de magnitud que se ejecutan en estos territorios son la recualificación urbana y readecuación de la zona de la antigua plaza de los Virreyes (plaza Túpac Amaru) y el parque Indoamericano, la política selectiva de mejoramiento y maquillaje urbano de conjuntos habitacionales como el Conjunto Habitacional Soldati y la inversión en algunos centros comerciales a cielo abierto como Chilavert (Villa Riachuelo) y Varela (Flores Sur).

Estos distintos abordajes de política corresponden a diversas zonas del sur de la Ciudad Autónoma de Buenos Aires, aunque este esquema debe ser matizado en el caso de la Villa 21-24, en la cual se implementan políticas que hemos denominado de gobernabilidad y está dentro del barrio de Barracas. También constituye una excepción al caso de Villa Riachuelo, donde se han adelantado obras de mejoramiento de espacio público ("puesta en valor" de la plaza Sudamericana, por ejemplo), lo que da lugar a un incipiente, pero veloz, desarrollo inmobiliario.

Otras políticas son transversales a los barrios, como la implementación del denominado Metrobús Sur, que conecta las zonas objeto de políticas.

\section{Palabras finales}

Luego del análisis de las iniciativas y prácticas realizadas por el macrismo en la zona sur de la ciudad, podemos sostener que esta gestión plantea una política neoliberal de ciudad, pero combinada, de forma contradictoria, con proyectos de mejoramiento del espacio público en zonas de sectores populares, que inclusive tienen mayor alcance que las gestiones anteriores. La diferencia con las acciones precedentes en barrios populares radica en que se trata de un política de "maquillaje urbano", que busca cambiar solo aspectos simbólicos de estos espacios, emblemáticos de la pobreza urbana, sin modificar su condición de barrios estigmatizados, su altísimo hacinamiento y las precarias condiciones habitacionales y de infraestructura básica. Tiene una doble función: ganar legitimidad interna en estos barrios y mostrar a los sectores de clase media y media alta del resto de la ciudad que se trata de políticas de "inclusión", es decir, se realizan acciones de bajo costo y alto impacto simbólico y mediático.

En cambio, en la zona norte se promueve "el desarrollo urbano", o sea, acciones en los espacios privados bajo la lógica de los negocios, donde el Estado inclusive ha subastado tierra pública para la construcción de vivienda de lujo. En síntesis, gobernabilidad e iniciativas de recualificación en la zona sur y facilitación del mercado en la zona norte. De esta forma, no se rompe la diferenciación cada vez más acentuada entre ambas zonas, sino que propone una construcción urbana, social y política diferenciada.

Afirmamos que estamos en los umbrales de una nueva etapa de despliegue del macrismo en la ciudad, en la que la implementación de obras de importante magnitud en el sur de la ciudad, la articulación de una red territorial propia 
dentro de los sectores populares y la consolidación de cuadros técnicos y políticos con mayor capacidad política son elementos que pueden ayudar a llevar a cabo grandes transformaciones que significarán una consolidación de este modelo de ciudad. Si tomamos la Comuna 8 como caso de análisis, se concluye que la articulación entre la remodelación del parque Indoamericano, la ampliación de la experiencia del Metrobús, el avance del polo farmacéutico, la reurbanización del algunas villas pequeñas y la aplicación de maquillaje urbano en otras, la construcción de un centro de cargas y transferencias, la readecuación del Microestadio de parque Roca y el parque de la Ciudad podrían provocar profundas transformaciones de las zonas donde vive la población más vulnerable de la Ciudad Autónoma de Buenos Aires, con lo que se acentúa la lógica expulsora de los sectores populares.

\section{Bibliografía}

Cosacov, N., Di Virgilio, M., Gil, A., Gil y De Anso, M., Guevara, T., Imori, M., Menazzi, M., Ostuni, F., Perea, C., Perelman, M., Ramos, J., Rodríguez, M., Paschkes, M., Vitale, P. (2011). Barrios al sur: Villa Lugano, Villa Riachuelo, Mataderos, Parque Patricios y Villa Soldati a través del tiempo. Documento de trabajo No. 56, Instituto Gino Germani, Universidad de Buenos Aires, Argentina.

Cosacov, N. y Faierman, E. (2012). Politicas públicas, rentas privadas. Politica habitacional y mercado inmobiliario en la Ciudad de Buenos Aires. Manuscrito presentado para su publicación.

Cravino, M. (2007). Transformaciones estructurales de las villas de emergencia. Despejando mitos sobre los asentamientos informales de Buenos Aires. Café de las Ciudades, 6(56).
Recuperado de http://www.cafedelasciudades. com.ar/tendencias_56_1.htm

Cravino, M., Ricciardi, M. y Sehtman, A. (2011). De la programación a la administración: avatares de la política de villas del macrismo (2007-2011). Manuscrito presentado para su publicación.

Di Virgilio, M. M. y Rodríguez, C. (2013). Producción social del hábitat. Buenos Aires: Café de las Ciudades.

Girola, M. F. (2005). Experiencias del lugar en un gran conjunto habitacional de la Ciudad de Buenos Aires: del proyecto moderno a la relegación urbana. Kairos. Revista de Temas Sociales, año 9(16). Recuperado de http://www.revistakairos. org/k16-archivos/Microsoft\%20Word\%20-\%20 Florencia\%20Girola.pdf. Pp 1-14.

Harvey, D. (2008). Breve historia del neoliberalismo. Madrid: Akal.

Herzer, H. (org.). (2008). Con el corazón mirando al sur. Transformaciones en el sur de la Ciudad de Buenos Aires. Buenos Aires: Espacio Editorial.

Instituto de Estadísticas y Censos. (2010). Censo Nacional de Población y vivienda. Buenos Aires: Autor.

Marcuse, P. (2004). Enclaves sí, guetos no: la segregación y el Estado. Revista de Estudios Regionales y Urbanos-Espacios y Debates (45), 24-33.

Oszlak, O. (1980). Políticas públicas y regímenes políticos: reflexiones a partir de algunas experiencias latinoamericanas. Documento de estudios CEDES, 3(2).

Perelman, M. (agosto de 2011). Sobre los modos legítimos de acceso al suelo urbano: el caso de la toma del parque Indoamericano. Conferencia 
presentada en el Primer Congreso Latinoamericano de Estudios Urbanos, Los Polvorines. Universidad Nacional de General Sarmiento, Argentina.

Theodore, N., Peck, J. y Brenner, N. (2009). Urbanismo neoliberal: la ciudad y el imperio de los mercados. Temas Sociales (66), 1-11
Vommaro, G. (mayo-junio de 2013). El estudio del reclutamiento de los dirigentes de una organización politica a través de sus generaciones politicas: el caso del Pro en la Ciudad Autónoma de Buenos Aires. Conferencia presentada en el Congreso LASA. Washington, entidad que organizó el evento. 